\title{
Analyse de la flore des macroalgues de trois sites marins de Dakar (PNIM, Soumbédioune et Terrou-bi) au Sénégal
}

\author{
Modou fall GUEYE*, Moussa yagame BODIAN, Mame Samba MBAYE, Godar SENE et \\ Kandioura NOBA
}

\author{
Laboratoire de Botanique et Biodiversité, Département de Biologie Végétale, Faculté des Sciences et \\ Techniques, Université Cheikh Anta DIOP, B.P.5005 Dakar-Fann, Sénégal. \\ *Auteur correspondant ; E-mail : gueyemodoufall@yahoo.fr; Tel: +221772317333.
}

\section{RESUME}

L'espace maritime du Sénégal est très vaste et renferme une riche biodiversité algale. Malgré cette grande étendue d'eau, ces macroalgues marines sont encore peu connues dans ce pays. Ce travail constitue une étude de la flore de macroalgues de trois sites marins de DAKAR. Elle a été entreprise pour connaitre la diversité spécifique, déterminer la structure de la flore (spectre taxonomique) et sa répartition par rapport à ces trois sites. Durant cette étude, des macroalgues ont été inventoriées, identifiées puis classées. Les résultats indiquent que cette flore est constituée de 21 taxons répartis dans 13 genres, 11 familles et 3 classes. Les classes des Ulvophyceae et des Florideophyceae sont les plus représentées, concentrant chacune 38,09\% des espèces rencontrées, suivies de celle des Phaeophyceae avec 23,8\% des espèces rencontrées. Cette étude a montré que la zone du PNIM (Parc National des Îles de la Madeleine) est plus riche en espèces avec 16 espèces $(55,1 \%)$, suivie de la zone de Terrou-bi avec 11 espèces $(37,9 \%)$ et de Soumbédioune avec 2 espèces $(6,8 \%)$. (C) 2019 International Formulae Group. All rights reserved

Mots clés: Flore, Macroalgues marines, PNIM, Soumbédioune, Terrou-bi, Sénégal.

\section{Systematic composition of macroalgae flora along Dakar Atlantic coast (NPMI, Soumbédioune and Terrou-bi), Senegal (West Africa)}

\begin{abstract}
In Senegal, a lot of botanical studies have been carried out on the phanerogamic flora but rarely on bryophytes and algae flora despite their ecological, economic and scientific importance. Therefore, the aim of this study was to determine the structure of macroalgae flora through systematic composition in three different sites which are National Park of Madeleine Island, Soumbédioune and Terrou-bi located along the Dakar coast. During this study, seaweeds were inventoried, conserved and identified. The results showed that flora consisted of 21 taxa distributed in 13 genera, 11 families and 3 classes. The classes with the highest species richness were Ulvophyceae and Florideophyceae with respectively $(38.09 \%)$ of recorded species while $23.8 \%$ of the flora belonged to the class of Phaeophyceae. This flora was more diverse in National Park of Madeleine Island where it was composed of 16 species $(55.1 \%)$, followed by Terrou-bi with 11 species $(37.9 \%)$ and the lowest number of species were recorded at Soumbédioune with only 2 species.
\end{abstract}

(C) 2019 International Formulae Group. All rights reserved

Keywords: Flora, Marine macroalgae, Atlantic coast, Senegal. 


\section{INTRODUCTION}

La zone littorale sénégalaise s'étend sur environ $700 \mathrm{~km}$. Du point de vue étendue, l'espace maritime représente environ 198.000 $\mathrm{km}^{2}$ (Diop et al., 2002).

Le Parc National des Iles de la Madeleine est situé dans cette zone à l'ouest de la presqu'île de Dakar. Ces îles sont protégées depuis 1949 et c'est en 1976 que le statut de parc national leur a été attribué. La façade continentale de ces îles correspond à la plage de Terrou-bi et à celle de Soumbédioune, situées sur la corniche ouest de Dakar. D'après Noba et al. (2003), le Parc National des îles de la Madeleine (PNIM) possède une flore très diversifiée composée de 107 espèces réparties dans 87 genres et 37 familles. Selon WWF (2011), le parc renferme également une faune bien représentée composée de 72 espèces dont 7 reptiles, 2 mammifères, 15 insectes et surtout de 48 oiseaux. Cependant, peu de données existent concernant la diversité des macroalgues. Pourtant, les travaux de Diedhiou (2010) ont révélé leur présence dans cette localité. Gueye et al. (2014) a révélé la présence de 38 taxa de microalgues marine dans l'île de Ngor.

Les macroalgues sont des organismes photosynthétiques marins dont l'appareil végétatif est un thalle c'est à dire sans tiges, ni racines, ni feuilles différenciées (Cabioc'h et al., 2006 ; Barry et al., 2014). Cependant, les organismes constituant le règne "végétal » se sont avérés aujourd'hui constituer un ensemble polyphylitique répartis dans 9 des 10 règnes du vivant, actuellement reconnus au sein des Eucaryotes. De même, ce qui est nommé « algues» est un ensemble d'organismes polyphylitiques répartis dans 8 des 10 règnes du vivant, 7 faisant partie des Eucaryotes et 1 des procaryotes (Boudouresque, 2015).

Les algues sont utilisées dans divers domaines : l'agro-alimentaire, l'industrie pharmaceutique, la cosmétologie et l'industrie de la peinture (Cabioc'h et al., 2006).

Malgré l'usage multivarié des macroalgues, peu d'études systématiques ont été publiées au Sénégal, entrainant ainsi une faible connaissance de leur diversité et de leur importance économique. En effet, les premières études sur les algues benthiques de la côte sénégalaise remontent vers les années 1930. Par la suite, les travaux de Dangeard (1952) sur la presqu'île du Cap Vert ont montré la présence d'un potentiel important d'algues sur les côtes de Dakar et de ses environs. Bodard et Mollion (1974) ont travaillé sur la végétation infralittorale de la petite côte sénégalaise à partir de 8 chalutages et 20 dragages. Les résultats de ces campagnes ont permis d'inventorier et de décrire 17 Chlorophyceae, 14 Phaeophyceae et 103 Rhodophyceae soit au total 134 espèces. Moellet-Nzaou (1994) a effectué l'étude chimiotaxonomique de douze chlorophyceae des côtes sénégalaises. Par ailleurs, Ndiaye (1995) a fait une étude sur des métabolites secondaires isolés d'organismes marins de la côte sénégalaise. Harper et Garbary (1997) ont signalé 242 espèces de macroalgues marines dans la «Petite côte». Bodian (2000) a fait des études portant sur la systématique et la biologie des macroalgues récoltées dans la zone incluant Dakar et la «petite côte ». Ces études systématiques ont permis de réactualiser la liste d'environ 260 espèces de macroalgues au Sénégal. Par la suite, les travaux de Diedhiou (2010) ont aussi contribué à la connaissance de la biodiversité des macroalgues du Sénégal récoltées dans les îles de Dakar et ont révélé la présence de 38 espèces.

Malgré toutes ces études, des efforts restent à faire dans le domaine de l'algologie à savoir l'exploration des fonds marins.

Ainsi, cette étude se propose de contribuer à une meilleure connaissance des macroalgues marines au Sénégal et spécifiquement de (i) d'inventorier les macroalgues rencontrées dans ces trois sites; (ii) déterminer la structure de la flore (spectre taxonomique) et sa répartition spatiale.

\section{MATERIEL ET METHODES}

\section{Présentation de la zone d'étude}

La zone d'étude comprend trois sites différents: Le Parc National des Îles de la Madeleine (PNIM), La plage de Terrou-bi et La plage de Soumbédioune (Figure 1). 


\section{Le Parc National des îles de la Madeleine (PNIM)}

D'après Lô et Maynart (1982), le Parc National des Îles de la Madeleine (14 ${ }^{\circ} 39^{\prime}$ $18,5^{\prime \prime}$ Nord et $17^{\circ} 28^{\prime} 09,7^{\prime \prime}$ Ouest) est formé d'un ensemble d'îles regroupant les "îles aux serpents" (17 ha), les "îles lougnes" (6 ha) et un espace océanique (22 ha) situées à l'ouest de la presqu'île de Dakar.

Le PNIM est le plus petit parc au Sénégal. Cet espace mixte est constitué de quelques îlots rocheux (les basanites) au relief tourmenté et d'un espace océanique très riche en espèces marines. Sur ses 45 ha (dont 23 ha de terre émergées), de nombreuses espèces végétales (Adansonia digitata, Tamarindus indica, Andropogon gayanus, Brachiara distichophylla, etc.) et animales (des oiseaux comme le phaéton à bec rouge, le milan noir, le faucon pèlerin, une grande colonie de cormorans, couleuvres sifflantes etc.) se disputent un coin de tranquillité dans ce sanctuaire si proche du tumulte dakarois. Par ailleurs, des milliers d'oiseaux marins ont fait de cette zone leur lieu de repos et de reproduction (MEPN, 1997).

\section{La plage de Soumbédioune}

Elle est aussi appelée le marché aux poissons de Soumbédioune, se trouvant dans la commune de Médina (Dakar). Cette plage se situe entre les (coordonnées géographiques $14^{\circ} 40,714^{\prime}$ Nord et $17^{\circ} 27,598^{\prime}$ Ouest) entre le "Magic land" et le cimetière de Soumbédioune. Elle est fréquentée soit par les pêcheurs, soit par les populations qui viennent pour se baigner. Le canal 4 de Gueule Tapée déverse ses eaux usées et nauséabondes sur une partie de cette plage.

Les pêcheurs de cette localité s'organisent autour d'une association dénommée Comités Locaux des Pécheurs (CLP) dont les trois sites-pilotes de pêches de la région de Dakar sont : Soumbédioune, Yéne et Bargny. Ils ont essentiellement orienté leurs initiatives autour de la gestion des mérous, particulièrement du mérou blanc.

\section{La plage de Terrou-bi}

Elle se situe entre la cour suprême et l'hôtel Terrou-bi sur la corniche ouest (coordonnées géographiques $14^{\circ} 41,036^{\prime}$ Nord et $17^{\circ} 27,796^{\prime}$ Ouest). C'est une plage publique très fréquentée par les baigneurs. Elle est caractérisée par la présence de beaucoup de roches où se fixent des macroalgues et d'autres espèces animales comme les mollusques, les méduses et les oursins.

\section{Récolte et Conservation des Macroalgues Prélèvement des échantillons}

Les échantillons de macroalgues ont été prélevés pendant des périodes de marée basse dont la hauteur au Sénégal varie de 0,20 à $0,5 \mathrm{~m}$. La récolte durant cette période permet d'explorer le maximum de surface côtière accessible à pied.

Les échantillonnages ont été effectués durant l'hivernage à partir du mois de juin jusqu'au mois de septembre 2014 qui correspond à la saison chaude en mer au Sénégal selon (Diaw et al, 1993). Deux récoltes mensuelles ont été effectuées dans chaque site, soit 8 sorties à intervalle irrégulier (06 juin, 21 juin, 02 juillet, 18 juillet, 04 août, 21 août, 05 septembre, 20 septembre 2014). Pendant le mois de septembre les fonds marins ont été explorés par plongée sous-marine avec l'aide technique des gardes côtes.

Les échantillons d'algue collectés ont été mis dans un sachet avec toutes les informations observables sur le terrain concernant son écologie et sa biologie (date, heure du jour de récolte, description générale de l'habitat, position au niveau du littoral, degré d'exposition à l'action des vagues, niveau de fixation sur le rocher, plantes ou animaux associés, etc.).

\section{Identification des échantillons}

L'identification des échantillons a commencé sur le terrain et s'est poursuivie au laboratoire. Elle est essentiellement basée sur l'observation à l'œil nu, à la loupe ou au microscope optique. 
L'observation à l'œil nu permet de relever tous les caractères morphologiques les plus "accessibles" telle que la couleur (pigment), la nature du thalle, la forme et l'amplitude de la ramification du thalle, etc.

L'observation à la loupe ou au microscope permet de voir les caractères fins comme les organes sexués et asexués, les rhizines, la structure du thalle.

Dans ce travail, l'identification a été basée sur l'observation des caractères morphologiques en comparant nos données à certains travaux comme ceux de Dangeard (1952), Gayral (1958) Bodard et Mollion (1974) Harper et Garbary (1997), Bodian (2000), De Reviers (2002, 2003), GaronLardiere (2004), Cabioc'h et al. (2006), Diedhiou (2010), Diaz-mur et Igniacio (2014) tout en se basant sur les critères suivants : le type de ramification, la couleur, la consistance, les organes de fixation, l'aspect du thalle.

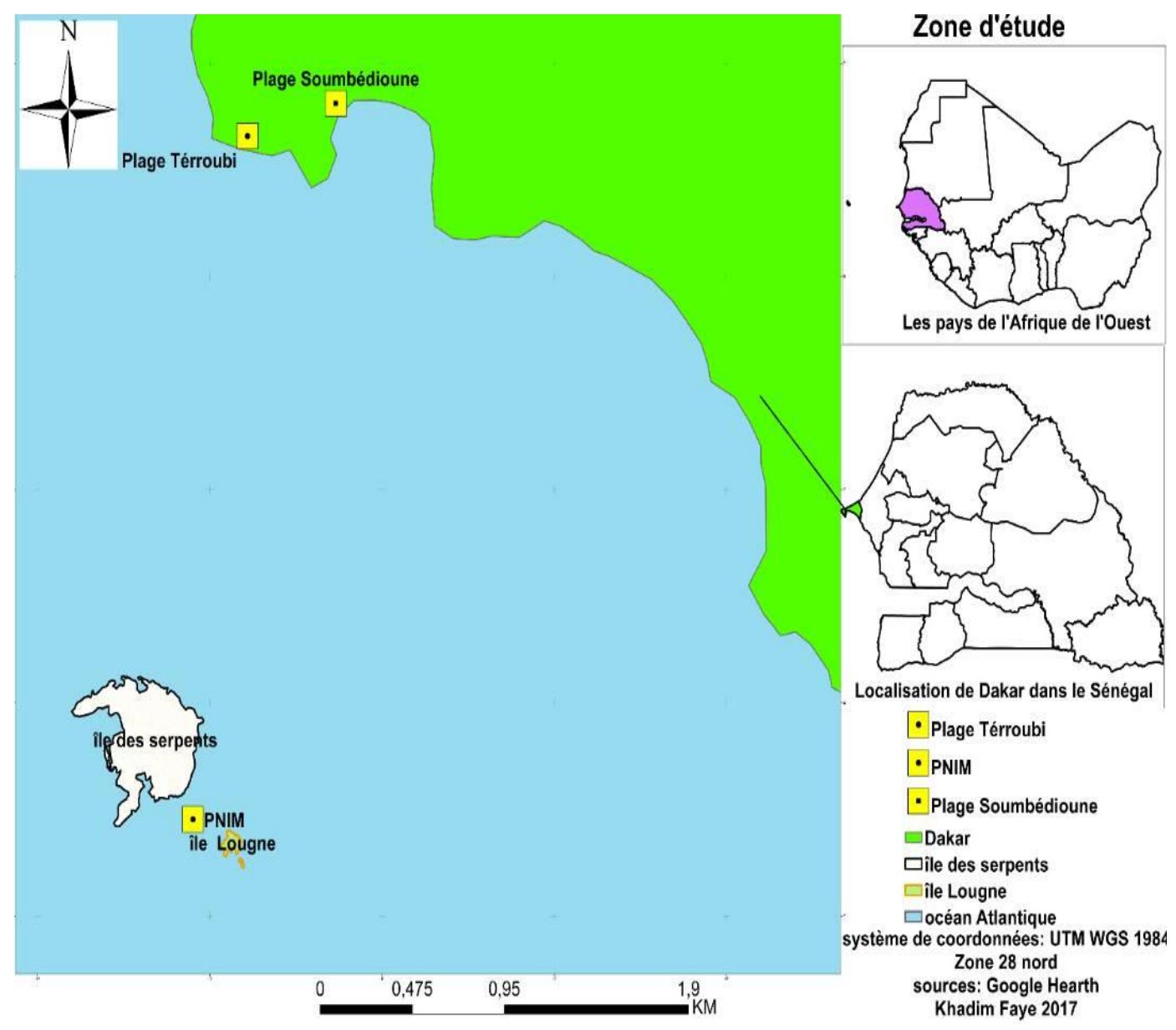

Figure 1 : Carte de la zone d'étude (PNIM, Soumbédioune, Terrou-bi). 


\section{RESULTATS}

\section{Structure de la flore des macroalgues et spectre taxonomique des macroalgues des sites étudiés}

L'observation des différents échantillons a permis d'identifier au niveau des trois sites 21 taxons de macroalgues appartenant à 13 genres, 11 familles et 3 classes (Ulvophyceae, Phaeophyceae et Florideophyceae). En terme de nombre de taxa, la classe des Florideophyceae et celle des Ulvophyceae sont les plus représentées avec chacune 38,09\% des espèces rencontrées. Celle des Phaeophyceae est faiblement représentée avec 23,8\% des espèces rencontrées. Les familles des Ulvaceae, Gelidiaceae et des Dictyotaceae sont plus représentées avec chacune $14,3 \%$ des familles suivi de celle des Bryopsidiaceae, Cladophoraceae, Corallinaceae et des Rhodomelaceae $9,52 \%$ chacune et enfin les familles Caulerpaceae, Scytosiphonaceae, Sargassaceae, Caulacanthaceae sont les plus faiblement représentées avec chacune 4,76\% (Tableau 1). La zone du PNIM domine les effectifs avec 16 espèces soit $55,1 \%$, suivie de la zone de Terrou-bi avec 11 espèces soit $37,9 \%$ et enfin de la zone de Soumbédioune avec 2 espèces soit 6,8\%. (Tableau 3 )

Le Tableau 2 montre plus de détails quant à la structure des macroalgues des sites PNIM, Terrou-bi et Soumbédioune. \% F.C.: Pourcentage des Familles par Classe; \% G.C.: Pourcentage des Genres par Classe

\section{La Répartition des macroalgues rencontrées en fonction des sites}

L'ensemble des espèces récoltées lors de cette étude sont consignées dans le Tableau 3 en fonction de leur site de récolte.

A Terrou-bi, 11 espèces différentes ont été recensées: 4 Chlorophycées, 3 Phéophycées et 4 Rhodophycées. A Soumbédioune, 2 espèces ont été trouvées : une Phéophycée et une Rhodophycée. Au PNIM, 16 espèces ont été inventoriées : 6 Chlorophycées, 2 Phéophycées et 8 Rhodophycées.

Les résultats enregistrés montrent qu'il n'y a pas d'espèces communes aux 3 sites. Cependant, les espèces suivantes : Laurencia pinnatifida, Corallina elongata, Corallina officinalis, Caulacanthus ustulatus, Dictyopteris delicatula, Ulva sp et Chaetomorpha antennina sont rencontrées aussi bien au PNIM qu'au Terrou-bi. L'espèce Gelidium pusillum est la seule rencontrée à Soumbédioune et au PNIM. A côté de ces groupes, des espèces ont été rencontrées dans un seul site, au Terrou-bi : Bryopsis plumosa, Caulerpa racemosa, Colpomenia sinuosa, Padina gymnospora uniquement rencontrées.

Pour les espèces : Bryopsis balbisiana, Chaetomorpha linum, Ulva lactuca, Ulva rigida, Dictyota ciliolata, Gelidium latifolium, Gelidium sesquipedale, Laurencia obtusa, elles ont été rencontrées seulement au PNIM. La Phaeophyceae: Sargassum vulgare a été récoltée en épave uniquement à Soumbédioune. 
Tableau 1 : Structure taxonomique des macroalgues des sites PNIM, Soumbédioune et Terrou-bi.

\begin{tabular}{|c|c|c|c|c|c|c|}
\hline \multirow{2}{*}{ Espèces } & \multicolumn{2}{|l|}{ Classes } & \multirow{2}{*}{ Familles } & \multirow{2}{*}{$\begin{array}{l}\% \\
\text { E.F }\end{array}$} & \multirow{2}{*}{ N.E.G } & \multirow{2}{*}{ N.G.C } \\
\hline & \multirow{6}{*}{ Nom } & $\%$ E.C & & & & \\
\hline Bryopsis balbisiana Lamouroux & & \multirow{9}{*}{38,09} & \multirow{2}{*}{ Bryopsidiaceae } & \multirow{2}{*}{9,52} & \multirow{2}{*}{2} & \multirow{9}{*}{4} \\
\hline Bryopsis plumosa (Hud.) C. Agardh & & & & & & \\
\hline $\begin{array}{lll}\text { Caulerpa } & \text { racemosa } & \text { peltata } \\
\text { (Lamouroux) } & & \\
\end{array}$ & & & Caulerpaceae & 4,76 & 1 & \\
\hline $\begin{array}{l}\text { Chaetomorpha antennina (Bory de S- } \\
\text { V) Kützing }\end{array}$ & & & \multirow{3}{*}{ Cladophoraceae } & \multirow{3}{*}{9,52} & \multirow[b]{3}{*}{2} & \\
\hline Chaetomorpha linum (O.F.Müller) & & & & & & \\
\hline Kützing & & & & & & \\
\hline \multicolumn{5}{|l|}{ Ulva rigida C.Agardh } & & \\
\hline Ulva lactuca Linnaeus & & & Ulvaceae & 14,3 & 3 & \\
\hline \multicolumn{5}{|l|}{ Ulva sp } & & \\
\hline $\begin{array}{lll}\text { Colpomenia sinuosa } & \text { (Mertens ex } \\
\text { Roth) Derbés\& Solier } & & \\
\end{array}$ & \multirow{5}{*}{ 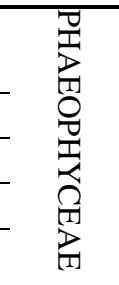 } & \multirow{5}{*}{23,8} & Scytosiphonaceae & 4,76 & 1 & \multirow{5}{*}{5} \\
\hline Dictyopteris delicatula Lamouroux & & & \multirow{3}{*}{ Dictyotaceae } & \multirow{3}{*}{14,3} & 1 & \\
\hline Dictyota ciliolata Sonder ex Kützing & & & & & 1 & \\
\hline Padina gymnospora (Kützing) Sonder & & & & & 1 & \\
\hline Sargassum vulgareC.Agarh & & & Sargassaceae & 4,76 & 1 & \\
\hline $\begin{array}{l}\text { Caulacanthus ustulatus (Mertens ex } \\
\text { Turner) Kützing }\end{array}$ & \multirow{8}{*}{ 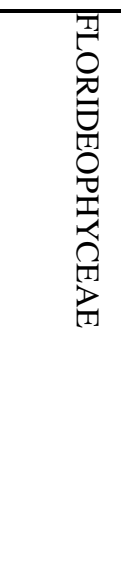 } & \multirow{8}{*}{38,09} & Caulacanthaceae & 4,76 & 1 & \multirow{8}{*}{4} \\
\hline Corallina elongata Ellis \&Solander & & & \multirow{2}{*}{ Corallinaceae } & \multirow{2}{*}{9,52} & \multirow{2}{*}{2} & \\
\hline Corallina officinalis Linnaeus & & & & & & \\
\hline $\begin{array}{l}\text { Gelidium sesquipédale (Clemente) } \\
\text { Thuret }\end{array}$ & & & & & & \\
\hline $\begin{array}{l}\text { Gelidium pusillum (Stackhouse) Le } \\
\text { jolis }\end{array}$ & & & Gelidiaceae & 14,3 & 3 & \\
\hline Gelidium latifolium Bornet ex Hauck & & & & & & \\
\hline Laurencia obtusa (Hud.) Lamouroux & & & \multirow[b]{2}{*}{ Rhodomelaceae } & \multirow[b]{2}{*}{9,52} & \multirow[b]{2}{*}{2} & \\
\hline $\begin{array}{lll}\text { Laurencia } & \text { pinnatifida } & \text { (Hud.) } \\
\text { Lamouroux } & & \\
\end{array}$ & & & & & & \\
\hline
\end{tabular}

Tableau 2 : Structure de la flore des macroalgues récoltées.

\begin{tabular}{lllllll}
\hline \multirow{2}{*}{ Classes } & Familles & \multicolumn{3}{c}{ Genres } & \multicolumn{3}{c}{ Espèces } \\
\cline { 2 - 7 } & Nombre & \% F.C & Nombre & \% G.C & Nombre & \% E.C \\
\hline Ulvophyceae & 4 & 36,36 & 4 & 30,76 & 8 & 38,09 \\
Phaeophyceae & 3 & 27,27 & 5 & 38,46 & 5 & 23,8 \\
Florideophyceae & 4 & 36,36 & 4 & 30,76 & 8 & 38,09 \\
Total & 11 & $100 \%$ & 13 & $100 \%$ & 21 & $100 \%$ \\
\hline
\end{tabular}


Tableau 3 : Répartition des macroalgues en fonction des sites. Le signe $(\times)$ signifie la présence de l'espèce dans le site.

\begin{tabular}{|c|c|c|c|}
\hline \multirow{2}{*}{ ESPECES } & \multicolumn{3}{|l|}{ SITES } \\
\hline & TEROU-BI & SOUMBEDIOUNE & PNIM \\
\hline Bryopsis balbisiana Lamouroux & & & $x$ \\
\hline Bryopsis plumosa (Hud.) C.Agarh & $x$ & & \\
\hline Caulerpa racemosa v.peltata Lamouroux & $x$ & & \\
\hline Chaetomorpha antennina(Bory) Kützing & $x$ & & $x$ \\
\hline Chaetomorpha linum (O.F. Müller) Kützing & & & $x$ \\
\hline Ulva sp & $x$ & & $x$ \\
\hline Ulva lactuca Linnaeus & & & $x$ \\
\hline Ulva rigida $\mathrm{C}$. Agardh & & & $x$ \\
\hline Colpomenia sinuosa (Mertens ex Roth) Derbés \& Solier & $x$ & & \\
\hline Dictyopteris delicatula Lamouroux & $x$ & & $x$ \\
\hline Dictyota ciliolata Sonder ex Kützing & & & $x$ \\
\hline Padina gymnospora (Kützing) Sonder & $x$ & & \\
\hline Sargassum vulgareC.Agarh & & $x$ & \\
\hline Caulacanthus ustulatus (Mertens ex Tuner) Kützing & $x$ & & $x$ \\
\hline Corallina elongata Ellis \& Solander & $x$ & & $x$ \\
\hline Corallina officinalis Linnaeus & $x$ & & $x$ \\
\hline Gelidium latifoliumBornet ex Hauck & & & $x$ \\
\hline Gelidium pusillum (Stackhouse) Le jolis & & $x$ & $x$ \\
\hline Gelidium sesquipedale (Clemente) Thuret & & & $x$ \\
\hline Laurencia obtusa (Hud.) Lamouroux & & & $x$ \\
\hline Laurencia pinnatifida (Hud.) Lamouroux & $x$ & & $x$ \\
\hline Total & 11 & 2 & 16 \\
\hline
\end{tabular}

\section{DISCUSSION}

D'après les résultats obtenus, 21 taxons ont été représentées. Un total de 20 taxons sont identifiés dans les trois sites d'étude jusqu'au niveau espèce plus une jeune ulve indéterminée.

La classe des Rhodophyceae et celle des Ulvophyceae sont plus représentées avec chacune 08 espèces, 04 Genres et 04 Familles. La classe des Phaeophyceae est la plus faiblement représentée avec 5 espèces réparties dans 3 familles et 5 genres. Ces résultats sont en conformité avec les travaux de Diedhiou (2010) qui a rencontré un plus faible nombre de phaeophyceae. Un résultat similaire a également été rapporté par Bodian (2000) qui trouve 50\% de Rhodophyceae, $36 \%$ de Chlorophyceae et $16 \%$ de phaeophyceae. En revanche, nos résultats contredisent ceux de Gervasoni et Ritter (2012) de même que ceux rapportés par
Person (2011). Ces auteurs estiment qu'il y'a un plus faible nombre d'espèces de Chlorophytes dans le monde.

Ainsi, 11 espèces ont été rencontrées au Terrou-bi, 2 espèces à Soumbédioune et 16 espèces au PNIM. Cette répartition inégale pourrait être liée à l'action anthropique qui affecte les zones d'étude. En effet la plage de Soumbédioune est caractérisée par la forte présence des pêcheurs et les eaux usées déversées par le canal de gueule tapée. Ceci pourrait partiellement expliquer la faible présence des macroalgues (Mama, 2011). Tous les taxons de cette collection ont été déjà signalés par des études antérieurs Bodian (2000); Diedhiou 2010) effectuées sur le littoral atlantique sénégalais.

Diedhiou (2010) a décrit 16 espèces dans ce parc national des îles de la Madeleine durant une partie de la saison sèche (mars, avril et mai). Les espèces Ulva fasciata, 
Enteromorpha $s p$ et Grateloupia doryphora qui figurent dans sa collection n'ont pas été rencontrées au cours de cette présente étude. Par ailleurs, Chaetomorpha sp, Bryopsis balbisiana, Dictyopteris delicatula et Dictyota ciliolata absentes de la collection (Diedhiou, 2010) ont été récoltées lors de cette présente étude. Selon Robuchon (2014); Sankare et al. (2016) les communautés de macroalgues diffèrent à la fois en fonction des saisons mais aussi des sites.

\section{Conclusion}

Cette étude sur l'inventaire et la classification des macroalgues marines du Sénégal, effectuée entre juin et septembre 2014 dans le parc national des îles de la Madeleine (PNIM) et dans sa façade continentale à savoir, les plages de Soumbédioune et Terrou-bi, a permis de connaître la diversité spécifique pendant la saison des pluies, de déterminer la structure de la flore et sa répartition spatiale. Au total 21 taxons répartis dans 3 classes ont été identifiés. Il s'agit de la classe des Ulvophyceae, de la classe des Florideophyceae et de celles des Phaeophyceae. Les familles Ulvaceae, Gelidiaceae et Dictyotaceae sont plus représentées avec chacune $14,3 \%$ des familles suivi de Bryopsidiaceae, Cladophoraceae, Corallinaceae et de Rhodomelaceae $9,52 \%$ et enfin les familles Caulerpaceae, Scytosiphonaceae, Sargassaceae, Caulacanthaceae sont les plus faiblement représentées avec chacune $4,76 \%$ des familles. La classe des Florideophyceae et celle des Ulvophyceae sont les plus représentées avec chacune $38,09 \%$ des espèces rencontrées. Celle des Phaeophyceae est faiblement représentée et ne compte que $23,8 \%$ des espèces rencontrées. La zone du PNIM domine les effectifs avec 16 espèces soit $55,1 \%$, suivie de la zone de Terrou-bi avec 11 espèces soit $37,9 \%$ et enfin de la zone de Soumbédioune avec 2 espèces soit $6,8 \%$.

\section{CONFLIT D'INTERETS}

Les auteurs déclarent qu'ils n'ont pas de conflits d'intérêts.

\section{CONTRIBUTIONS DES AUTEURS}

GMF : Conception, collecte des données de terrain et rédaction de l'article. BMY : Lecture et correction de l'article. MMS : Lecture et correction de l'article. SG : Lecture et correction de l'article. NK : Lecture et correction de l'article.

\section{REMERCIEMENTS}

L'équipe scientifique remercie sincèrement la direction du Parc National des Îles de la Madeleine pour avoir facilité l'accès au parc.

\section{REFERENCES}

Barry M, Ouedraogo M, Sourabie S, Guissou PI. 2014. Intérêt thérapeutique de la spiruline chez l'homme: revue général. Int. J. Biol. Chem. Sci., 8(6): 2740-2749.

Bodard M, Mollion J. 1974. La végétation infralittorale de la petite côte Sénégalaise. Soc. Phycol. De France, Bull. $\mathrm{N}^{\circ} 19$.

Bodian MY. 2000. Systématique et biologie des algues macrophytes collectées pendant la période hivernale dans la zone incluant Dakar et la petite côte (Août et décembre 1998), Thèse de DEA, Faculté des Sciences et Techniques, Université Cheikh Anta Diop de DAKAR, p.162.

Boudouresque CF. 2015. Manuel de rédaction scientifique et technique. Edition 20142015, p.91. Cabioc'h J, Floc'hJ-Y, Le Toquin A, Boudouresque CF, Meinesz A,Verlaque M. 2006. Guide des Algues des mers d'Europe. Delachaux et Niestlé SA : Paris, p.271.

Dangeard P. 1952. Algues de la presqu'île du Cap Vert: Dakar et ces environs. Les Botanistes. Série XXXVI, Fascicule I. VI

De Reviers B. 2002. Biologie et Phylogénie des Algues (Tome 1). Editions Belin Sup. : Paris.

De Reviers B. 2003. Biologie et Phylogénie des Algues (Tome 2). Collection Belin Sup Science : Paris.

Diaw AT, Bâ A, Bouland P, Diouf PS, Lake L-A, Mbow M-A, Ndiaye, Thiam MD. 1993. Gestion des Ressources Côtières et Littorales du Sénégal: Actes de l'Atelier 
de Gorée 27-29 Juillet 1992. UICN, Gland, Suisse, $x+484$ pp.

Diaz-mur P, Ignacio B. 2014. Algues des roches couvertes de sable de la péninsule ibérique de l'atlantique. Partie 2. Palmariales, Ceramiales (Hors Rhodomelaceae), Gelidiales, Gigartinales, Rhodymeniales et Scytothamnales. Espagne. Cryptogamie, Algologie, 35(2): 157-199.

Diedhiou I. 2010. Contribution à la connaissance de la biodiversité des algues macrophytes marines du Sénégal, cas des îles de Dakar: Gorée, Madeleines, Ngor et Yoff Tonghor. Mémoire de master II. Faculté des Sciences et techniques, Université Cheikh Anta Diop, DAKAR, p.42

Diop S. 2002. Les écosystèmes marins et côtiers de l'Afrique de l'Ouest : enjeux de leur gestion à long terme et de leur développement durable - Exemple du Sénégal, p.15

Gervasoni E, Ritter L. 2012. Diversification des élevages : Etude des potentialités de culture de macroalgue alimentaires à Languedoc-Roussillon CEPRALMAR.

Garbary DJ, Beverridge LF, Flynn AD, White KL. 2012. Population ecology of Palmaria palmata (Palmariales, Rhodophyta) from harvested and nonharvested shores on Digby Neck, Nova Scotia, Canada. Algae, 27(1): 33-42.

Garon-Lardiere S. 2004. Etude structurale des polysaccharides pariétaux de l'algue rouge Asparagopsis armata (Bonnemaisonniales). Thèse de Doctorat de l'Ecole doctorale des sciences de la matière, de l'information et du vivant. Université de Bretagne Occidentale, p.226.

Gayral P. 1958. Algue de la Côte Atlantique Marocaine. La Nature au Maroc; p.52.

Gueye M, Ba N, Bodian MY, Mbaye MS, Basséne C, Kane M, Noba K, Ngom A. 2014. Les Cyanophyceae marine de l'île de Ngor (Sénégal). Int. J. Biol. Chem. Sci., 8(1): 144-156.

Harper JT, Garbary DJ. 1997. Marine Algae of Northern Senegal: The flora and its
Biogeography. Botanica Marina, 40 : 129-138.

Lô M, Maynart G, 1982. Contribution de la flore et de la végétation des îles de la Madeleine (îles aux serpents). Mémoire de l'IFAN, 92 : 91-98.

Mama D, Chouti W, Alassane A, Changotade O, Alapini F, Boukary M. 2011. Etude dynamique des apports en éléments majeurs et nutritifs des eaux de la lagune de Porto-Novo (Sud Bénin). Int. J. Biol. Chem. Sci., 5(3): 1278-1293.

Ministère de l'Environnement et de la Protection de la Nature. 1997. Rapport national Biodiversité, Sénégal.

Moellet-Nzaou AR. 1994. Etude chimiotaxonomique de douze chlorophycées des côtes Sénégalaise, Thèse de Doctorat du $3^{\mathrm{em}}$ cycle, Faculté des sciences et Techniques, UCAD, DAKAR p.165.

Ndiaye I. 1995. Etude structurale, dégradation synthèse biométrique de métabolites secondaires isolés d'organisme de la côte sénégalaise. Proposition de nouveau processus biogénétiques. Thèse de Doctorat d'état ès Sciences physiques, Faculté des sciences et Techniques. UCAD, DAKAR, p. 170.

Person J. 2011. Algues, filières du futur. Livre turquoise, Adebiotech, p.161.

Robuchon M. 2014. Etude spatio-temporelle de la biodiversité des forêts de laminaires des côtes bretonnes par une approche intégrée de génétique des populations et d'écologie des communautés, Thèse de Doctorat, Ecologie et évolution, Muséum national d'histoire Naturelle, .134.

Sankaré Y, Komoe K, Aka KS, Fofie NBY, Bamba A. 2016. Répartition et abondance des Sargassum natans et Sarassum fluitans (Sargassaceae, Fucales) dans les eaux marines ivoiriennes (Afrique de l'ouest). Int. J. Biol. Chem. Sci., 10(4): 1853-1864.

WWF. 2011. Analyse de l'efficacité de gestion des aires protégées du Sénégal par la méthodologie Rappam. WWF WAMER. 